(RESEARCH ARTICLE)

\title{
Spectral analysis of pathological gait characteristics of patients suffering from Parkinson disease and Strokes
}

\author{
Dias Kariyawasam Majuwana Gamage Induni Uresha 1, ${ }^{*}$, Munasinghe Aruna ${ }^{2}$, Ravindra Sudath³, Rodrigo \\ Dinushiya Shamalee ${ }^{1}$ and Senanayake Bimsara ${ }^{3}$
}

\author{
${ }^{1}$ Department of Mathematics, University of Sri Jayewardenepura, Nugegoda, Sri Lanka. \\ ${ }^{2}$ District General Hospital, Gampaha, Sri Lanka. \\ ${ }^{3}$ Institute of Neurology, National Hospital of Sri Lanka.
}

Publication history: Received on 28 May 2020; revised on 21 June 2020; accepted on 23 June 2020

Article DOI: https://doi.org/10.30574/wjarr.2020.6.3.0177

\begin{abstract}
Strokes and Parkinson's disease (PD) are the current leading cause of severe, and long-term disability prevailing in Sri Lanka. Detection of gait characteristics has become an interesting field of biomechanics that provides useful information in the management of the above two diseases. The objective of the study presented in this paper is to establish a technique for the gait detection of Stroke and PD patients using Discrete Fourier Transforms (DFT). Twenty four patients with PD and Stroke and five healthy controls were made to perform a series of movements which were measured using Kinect (a motion detection sensor produced by Microsoft). All the participants were recruited from the National Hospital Sri Lanka (NHSL). The Kinect device provided x, y, z coordinates of the ankle, knee, and hip joints. Ankle, knee, and hip joint angle characteristics have been analyzed in the frequency domain, and abnormal gait of Stroke and PD patients has been identified using harmonic coefficients. Experimental results and analysis indicate that both Stroke and PD patients have significant amplitude values for the first few harmonics relative to healthy controls. This result conforms with the shorter stride length property of Stroke and PD patients. For therapeutic interventions to improve gait performances, consideration should be given to both the severity of the disease and the lower limb involvement of the disease. Results conclude that the proposed method can accurately visualize the abnormality in the gait of Stroke and PD patients with sufficient accuracy.
\end{abstract}

Keywords: Parkinson's Disease; Stroke; Discrete Fourier transform; Gait analysis; Severity; Lower limb involvement.

\section{Introduction}

In the recent past, non-communicable diseases (NCDs) have emerged as one of the leading health problems in the world with $71 \%$ of the mortality rate [https://www.who.int/news-room/fact-sheets/detail/noncommunicable-diseases, Last accessed on 09/03/2020]. Due to the aging population and due to some risk factors, including physical inactivity, tobacco use, harmful use of alcohol, and unhealthy diet, the burden of NCD has increased [1]. NCDs have incurred massive health, financial and economic costs in many countries throughout the world. Sri Lanka is a middle-income country where the majority of the population is living below the poverty line. According to the World Health Organization (WHO) statistics [https://www.who.int/beat-ncds/countries/sri-lanka/en/, Last accessed 10/03/2020], NCDs are responsible for more than three-quarters of deaths in Sri Lanka.

Neurological disorders are the most common cause of death in Sri Lanka among NCDs. [https://www.who.int/beatncds/countries/sri-lanka/en/, Last accessed 10/03/2020]. According to the ranking of the Institute of Health Metric and Evaluation, Stroke is the second-leading cause of death in Sri Lanka from 2007 to 2017 [http://www.healthdata.org/sri-lanka, Last accessed 10/03/2020]. In Sri Lanka, according to WHO statistics, Parkinson's mortality accounted for $0.08 \%$ of all deaths. Due to urbanization and life-changing behaviors, the risk of

\footnotetext{
${ }^{*}$ Corresponding author: Dias Kariyawasam Majuwana Gamage Induni Uresha
} 
Stroke increases. Both genetic and environmental factors have been shown to be risk factors for PD [2]. Living with a Stroke or with PD is a challenge because both affect one's movements. In fact, Strokes and PD are the leading causes of acute and chronic disability.

PD is often caused by a progressive loss of dopamine-producing cells in the brain [3]. Dopamine is a chemical messenger that controls one's mobility [4]. The absence of dopamine-producing cells in the brain eventually leads to loss of gait performances. In general, slowness, tremor, and freezing of gait can be identified as hallmark features of PD [5]. The stroke is defined by the World Health Organization as a clot or rupture of blood vessels that transport oxygen and nutrients to the brain. Stroke affects the patterns of kinematic, muscular, and muscle activity in patients and can often induce spasticity [6]. A wide variety of gait performance can be visualized from post-stroke.

Recognition of walking features has generated considerable interest in the field of rehabilitation. Numerous statistical researchers have attempted to quantify differences in the gait pattern of patients with PD and Stroke compared to healthy controls $[2,7,8]$. In both diseases, most studies document the spatiotemporal changes in the gait pattern. There is a clear link between human gait characteristics and medical conditions $[9,10]$. Disability is a common characteristic of both PD and Stroke disease. Therefore gait analysis is important in disease diagnosis and monitoring in Stroke and PD.

In this paper, we introduce a method to identify gait abnormalities of Stroke and PD patients compared to healthy controls using the Discrete Fourier Transform technique. Here, we have considered three joint angles: ankle joint angle, knee joint angle, hip joint angle. Therefore the main aim of the study was to quantify differences in the gait performances of these two diseases compared to healthy controls. The research further quantifies the differences in the gait characteristics of PD and Stroke diseases depending on their severity.

\section{Methodology}

\subsection{Participants}

A study sample of twenty-four Stroke and PD survivors participated in this study. The study includes twelve male patients with PD (age: $66.50 \pm 3.39$ years; height: $1.6679 \pm 0.0471 \mathrm{~m}$; weight: $61.17 \pm 5.28 \mathrm{~kg}$; body mass index $(\mathrm{BMI}): 21.86 \pm 116 \mathrm{~kg} / \mathrm{m} 2$ ) and twelve male patients with Stroke disease (age: $59.43 \pm 4.28$ years; height: $1.6437 \pm$ $0.0439 \mathrm{~m}$; weight: $67.14 \pm 5.27 \mathrm{~kg}$, BMI: $24.68 \pm 1.16 \mathrm{~kg} / \mathrm{m} 2$ ). The healthy controls were selected from the population of healthy elderly people. All participants were selected from the neurological ward of National Hospital Sri Lanka (NHSL).

Participants were excluded when they met the following criteria: (1) they had brain surgery in the past; (2) they had clinically significant comorbidities likely to affect gait including diabetes, rheumatic or orthopedic disease, other neurological problems, depression, as determined by history or clinical evaluation; (3) their BMI value greater than 30; (4) inability to communicate with each other; (5) visual or musculoskeletal defects; (6) unable to walk independently without any assistance or device for at least $3 \mathrm{~m}$.

\subsection{Data collection}

The study was carried out under the ethical guidelines of the NHSL. The purpose of the study was explained to each individual and provided written informed consent before participation, by following the ethical principles. To realize research aims, skeleton readings ( $\mathrm{x}, \mathrm{y}, \mathrm{z}$ coordinates of the joint points) were captured using the instrument Kinect version 2 device. To take enough data, participants were asked to complete four sequences of walking between the beginning and endpoints towards the sensor at their normal speed and on barefoot. Joint angles: ankle, knee, and hip were calculated using the collected $\mathrm{x}, \mathrm{y}, \mathrm{z}$ coordinates of the joint points.

\subsection{Mathematical motivation to the study}

Human gait is periodic. Therefore, one complete gait cycle was extracted for all angle data. For the analysis three joint angles were considered: ankle, knee, and hip. For example, the joint angle data are considered as follows, $x(n)=$ ankle joint angle $(n)$ where $n$ is the sample number. Similarly, the other two joint angles have been defined. To carry out the comparison, data points were interpolated up to $293(=\mathrm{N})$ data samples.

$$
X(n)=\sum_{k=0}^{N-1} x(k) \exp \left(-\frac{2 \pi k n}{N}\right)
$$




$$
x(n)=\frac{1}{N} \sum_{k=0}^{N-1} X(k) \exp \left(\frac{2 \pi k n}{N}\right)
$$

Here $X(k)$ represents the frequency domain function and $x(n)$ represents the time domain function. The amplitude function can be expressed as follows,

$$
|X(k)|=\sqrt{R^{2}(k)+I^{2}(k)}
$$

Where $R(k)$ and $I(k)$ are the real and imaginary parts of the $X(k)$ respectively. DFT was implemented using FFT, a useful time-saving algorithm for computing DFT for each joint angle [11]. A program was written by using the mathematical software MATLAB to calculate DFT. The harmonic analysis was used to carry out comparisons.

\section{Experimental results and discussion}

The Discrete Fourier Transform (DFT) is a technique widely used to study periodic signals. Our inspection is based on the amplitude spectrum of joint angles: ankle, knee, and hip data. This section analyzes joint angles data and provides a clear insight into the abnormality of PD and stroke patients. This would aid in the extraction of significant features and specific information that helps in the management. The low-frequency components of the amplitude spectrum provide sufficient information that is very useful for management.

\subsection{Comparison with healthy controls}

This section briefly describes how the abnormality of the gait cycles of patients who are suffering from PD and the Strokes are identified comparing with healthy controls.

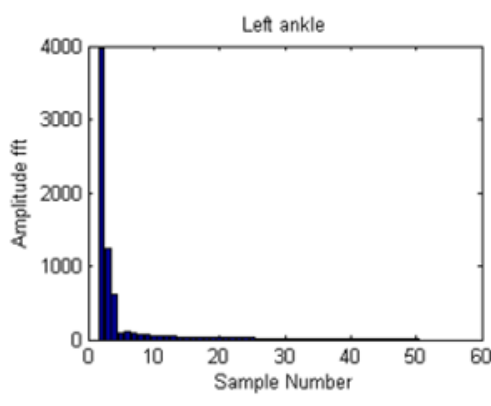

(a)

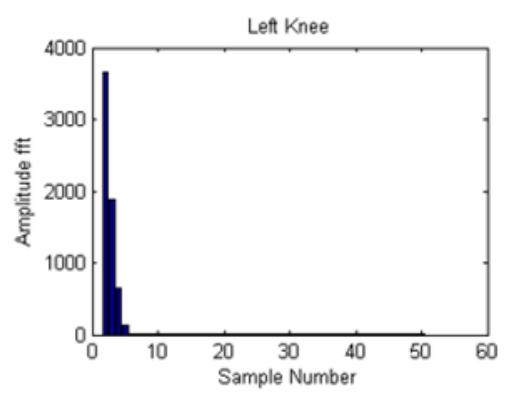

(b)

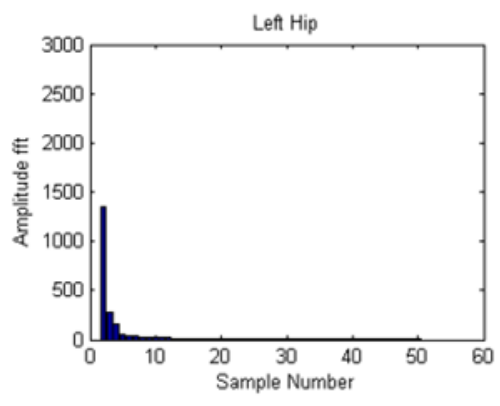

(c)

Figure 1 Amplitude spectrum of (a) ankle joint angle (b) knee joint angle (c) hip joint angle of healthy controls

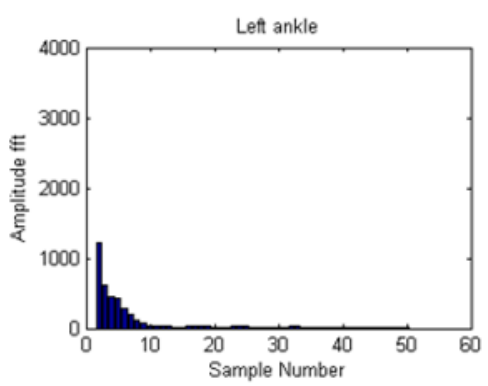

(a)

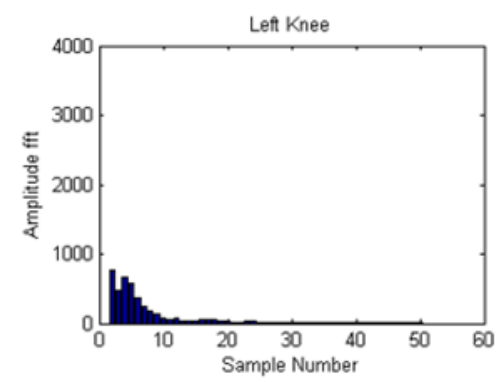

(b)

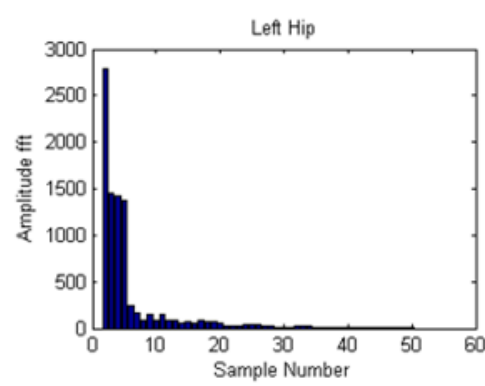

(c)

Figure 2 Amplitude spectrum of (a) ankle joint angle (b) knee joint angle (c) hip joint angle of Stroke patients 


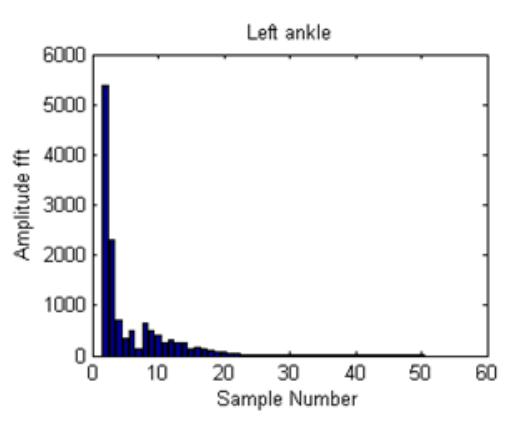

(a)

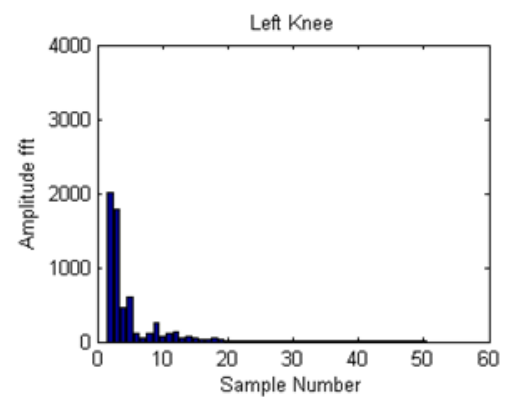

(b)

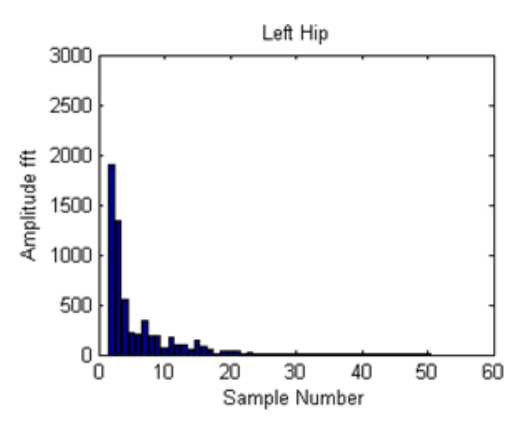

(c)

Figure 3 Amplitude spectrum of (a) ankle joint angle (b) knee joint angle (c) hip joint angle of PD patients

Figure 1 (a) shows significant amplitude values for the first three harmonics in the amplitude spectrum of the ankle joint angle of healthy controls gait whereas the amplitude spectrum of the ankle joint angle of the Stroke patient's gait (Figure 2(a)) shows significant values for the first ten harmonics. This implies that the stride length is shorter in Stroke patients compared to healthy controls. As shown in Figure 1(a) and Figure 2(a), the amplitude of the first harmonic in the amplitude spectrum of the ankle joint angle of Stroke patients is small compared to healthy controls. This indicates that there is an abnormality of the ankle joint in the patients with Stroke compared with healthy controls. Ankle joint and surrounding structures contribute to the balance of the body when walking. Therefore, due to spasticity occurs in the ankle joint muscles, limit the upward, downward movements of the foot referred to as dorsiflexion and plantar flexion. Reduction in knee flexion is a prominent feature of a Stroke patient [12]. As shown in Figure 1(b) and Figure 2(b), the amplitude of the first harmonic in the amplitude spectrum of the knee joint angle of Stroke patient's gait is lower compared to healthy controls. This illustrates that in Stroke patients, due to spasticity occurs in the lower limb muscles, knee flexion, and extension range drop off.

Since spasticity occurs in the lower limb muscles, the mobility of the hip joint as well as ankle and knee joints are limited. According to Figure 1(c) and Figure 2(c), the amplitude of the first harmonic in the amplitude spectrum of the hip joint angle of patients who are suffering from Stroke is higher than that of healthy controls. This is due to the spasticity occurs in the hip joint extensor and adductor muscles. As a result, the hip moves vertically and horizontally while walking. This is called circumduction gait, where the affected limb moves through an arc away from the body.

At the ankle joint, the amplitude spectrum of PD (Figure 3(a)) shows significant amplitude values for the first twenty harmonics, whereas the healthy control's amplitude spectrum of ankle joint angle (Figure 1(a)) shows significant amplitude values for the first three harmonics. This indicates the stride length of PD is much smaller compared to healthy controls. As shown, the amplitude of the first harmonic in the amplitude spectrum of the ankle joint angle of PD is higher (Figure 3(a)) compared to healthy controls (Figure 1(a)). This indicates that the entire foot of the patients with PD is placed on the ground at the same time. Since the stride-length is shorter in PD, those who have PD tend to walk slowly and drag their feet on the ground while walking.

According to Figure 3(b) and Figure 1(b), the amplitude of the first harmonic in the amplitude spectrum of the knee joint angle of PD is smaller compared to healthy controls. This indicates the bending posture of PD patients that limits the range of movability of the knee joint. The amplitude spectrum of the knee joint also demonstrates the shorter stride length property of PD patients. The notable feature that can be observed from Figure 3(c) is that the amplitude of the first harmonic is higher compared to Figure 1(c). This also indicates the abnormality of the PD patient's hip movement. Compared to the amplitude spectra of healthy controls, amplitude spectra of PD show fluctuations in the amplitude values that are due to the tremor of PD patients.

\subsection{Comparison according to the degree of severity}

The degree of severity of stroke disease depends on location of the blockage in the brain and the the extent of damage of brain tissues. If blockage is in the right side of the brain, then it causes nerve complications on left side of the body resulting in gait pattern abnormalities. Similar abnormalities in gait patterns have been observed among patients suffering from Parkinsonism. The decrease of Dopamine level which leads to increase of severity of the decreas0e is responsible for this. As both diseases cause abnormalities in gait patterns, details of such abnormalities can be utilized as a measure of their severities. 


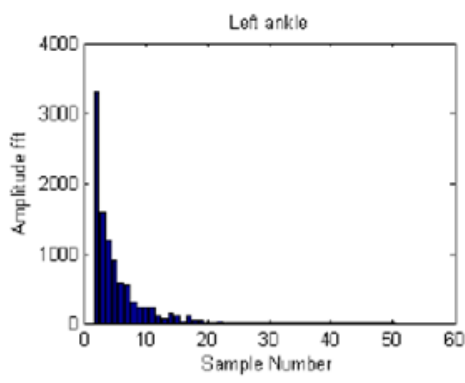

(a)

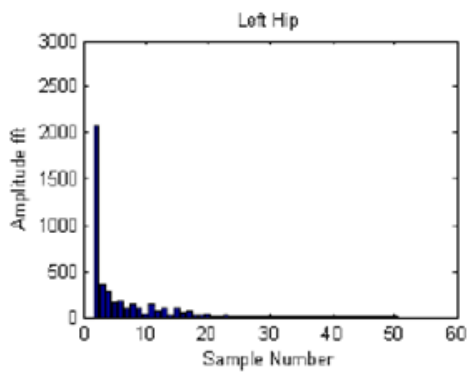

(d)

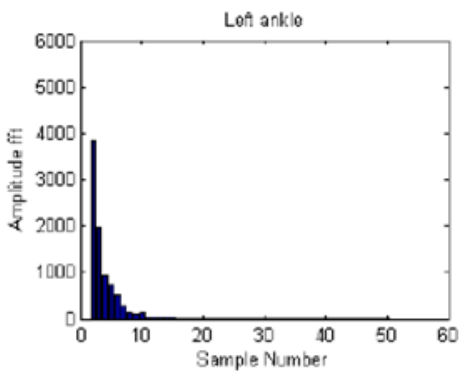

(g)

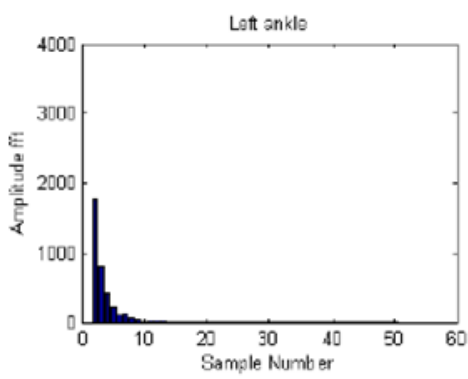

(b)

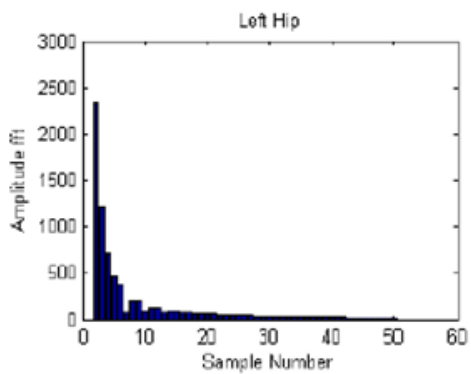

(e)

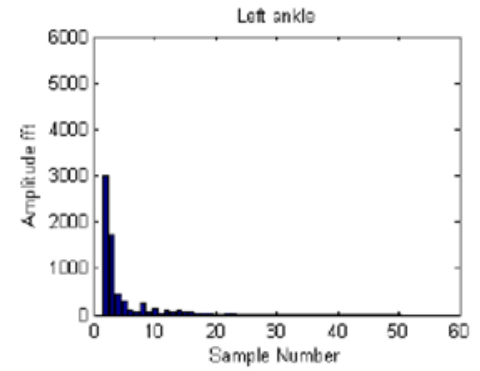

(h)

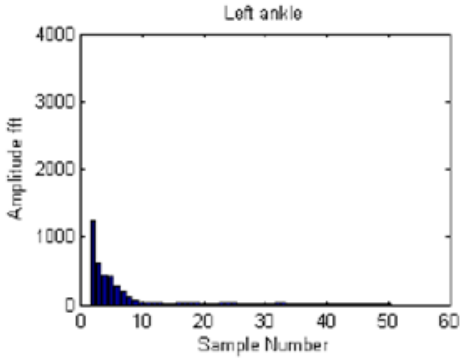

(c)

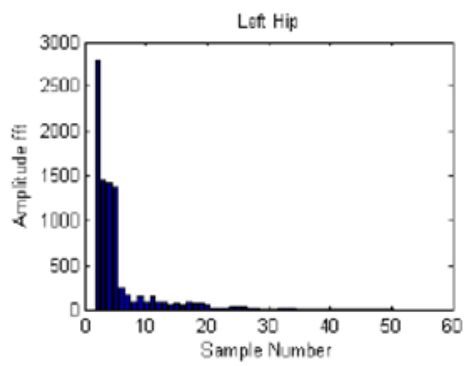

(f)

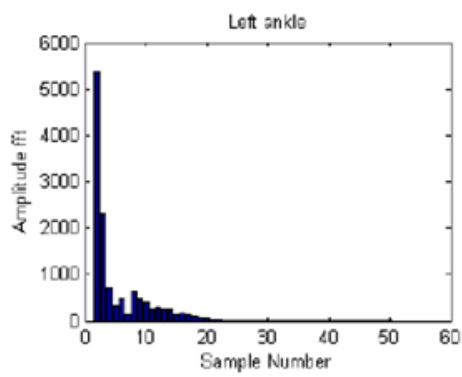

(i)

Figure 4 Amplitude spectrum of the Stroke and PD patient's gait angles according to the severity (a) left ankle joint angle of mild Stroke (b) left ankle joint angle of moderate Stroke (c) left ankle joint angle of severe Stroke (d) left hip joint angle of mild Stroke (e) left hip joint angle of moderate Stroke (f) left hip joint angle of severe Stroke (g) left ankle joint angle of mild PD (h) left ankle joint angle of moderate PD (i) left ankle joint angle of severe PD.

Figures 4(a), 4(b), and 4(c) show that the amplitude of the first harmonic of amplitude spectra of ankle joint angles of the Stroke patients decreases according to the degree of severity. This indicates that the level of the spasticity in the ankle joint muscles increases with increasing severity. This same pattern is obtained for the knee joint angle but amplitude spectrums of the hip joint angle according to severity show a distinguishing feature. i.e. the amplitude of the first harmonic increases with the severity (Figure 4(d)-Figure 4(f)).

The degree of dopamine loss correlates with the severity of PD. Unlike in Stroke disease, the amplitude spectra of patients with PD show a distinguishing feature with the severity. Figure 4(g), 4(h), and 4(i) show that the magnitude (height of the column) of the amplitudes of the amplitude spectra of the ankle joint increase with the severity as the shape of a bell. Both knee and hip joints show a similar pattern. This indicates that bell shape abnormality increases according to the degree of severity. This happens due to treatments given to patients with severe PD. Since all the participants are selected from a rehabilitation center, patients with PD may be in an onset medication.

\subsection{Identify lower limb involvement of both diseases}

For the explanation, the knee joint angle amplitude spectrum is used for both Stroke and PD to identify lower limb involvement. 

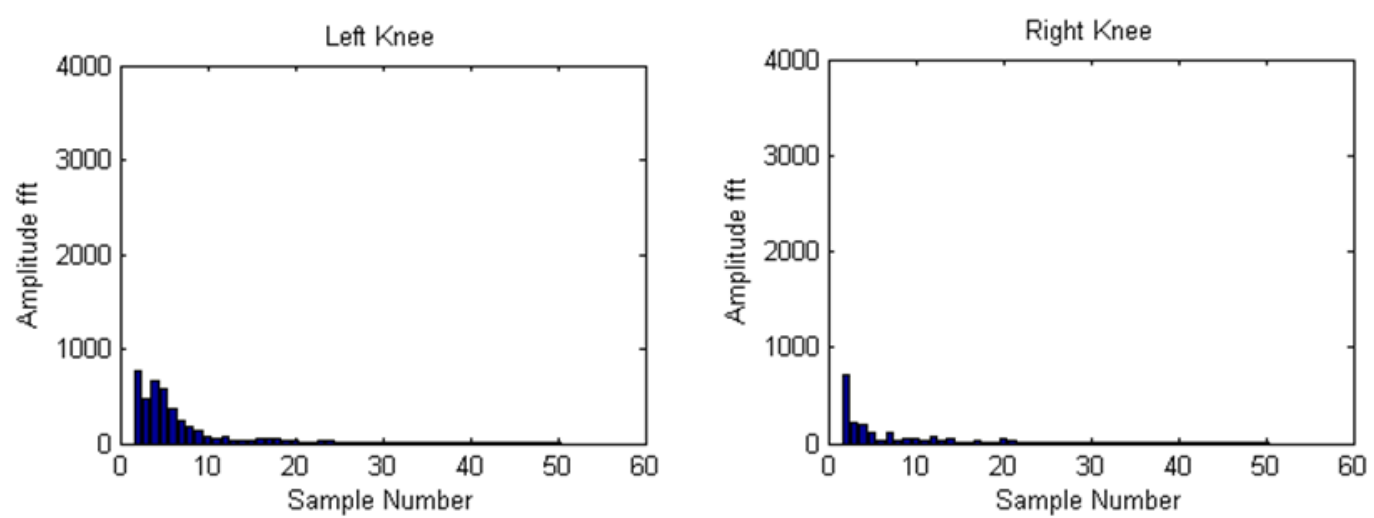

Figure 5Comparison between the amplitude spectra of the left and right sides of the Stroke patient's gait angles

As shown in Figure 5, significant amplitude values can be seen on both left and right sides, for the first ten harmonics. But the notable feature that can be seen from the amplitude spectrum of the left side is greater the magnitudes of the amplitudes compared to the right side. This indicates that the degree of abnormality is high on the left side. Therefore, experimental results confirm that the bodyweight of a patient with Stroke is asymptotically loaded into lower limbs. The distinct feature that can be observed from the amplitude spectra of the left and right sides is that the Stroke is mostly affected to one side.
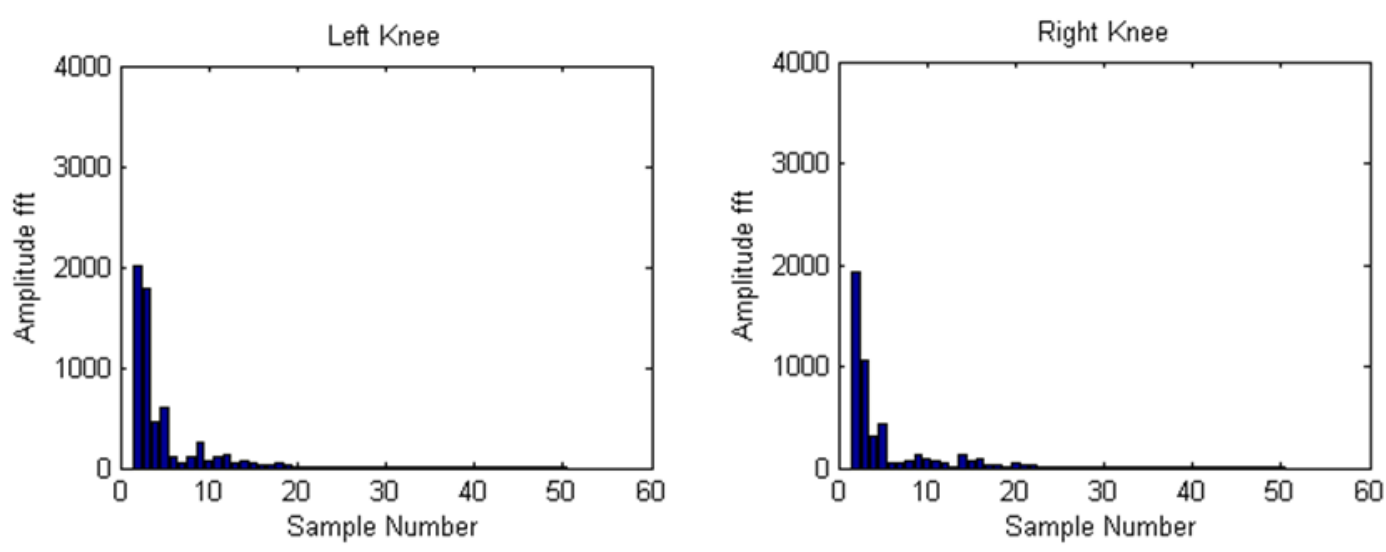

Figure 6 Comparison between the amplitude spectra of the left and right sides of the PD patient's gait angles.

According to Figure 6, the first twenty harmonics of the amplitude spectra of the left and right sides of the patients with PD show significant amplitude values. The distinct feature that can be observed from the amplitude spectrum of the left side compared to the right side is that magnitudes of the amplitude values are higher on the left side. This implies the degree of abnormality is high on the left side. Compared to Figure 5, Figure 6 does not show a notable difference between the left and right sides. This indicates the asymmetry of the affected and unaffected sides is more pronounced in patients with Stroke disease compared to PD.

\subsection{Comparison between Stroke disease and Parkinson's disease}

At the ankle joint, Figure 3(a) shows higher amplitude values from the first harmonic to the twenties, whereas Figure 2(a) shows only for the first ten harmonics. This indicates that the stride length of patients with PD is smaller compared to Stroke ones. Small shuffling steps can be seen in PD patients [13]. The amplitude of the first harmonic is high and fluctuations can be observed from Figure 3(a) compared to Figure 2(a). This shows the tremor and flat foot strike characteristic of patients with PD compared to Stroke. In addition to ankle joint, knee joint also behaves similarly but hip joint shows a special feature. i.e. amplitude of the first harmonic (Figure 3(c)) at PD is less relative to the Stroke (Figure 3(c)). This indicates the bent posture of PD patients. Compared to the gait of patients with PD, Stroke disease patients show a special gait called circumduction gait that is due to the spasticity occurring in the hip joint muscles. 


\section{Conclusion}

This paper provides an approach to abnormal gait identification using a DFT analysis. The experimental results indicate ankle, knee, and hip joint angles in the frequency domain can be used to characterize the abnormality and to identify special characteristics of these two diseases with the severity.

In Stroke disease, unlike in the ankle and knee joint angle, the hip joint angle shows a special feature called a circumduction gait feature. The results indicate that the stride lengths of PD and Stroke are smaller compared to healthy controls. This technique visualizes the gradual increment and bell-shaped increment of the abnormality of patients with Stroke and PD respectively according to the severity. The amplitude value of the first harmonic also illustrates the deviation of the gait of the PD and the Stroke according to their severity. The results show that the stride length of PD and Stroke is smaller compared to healthy controls. Patients with PD characterized a smaller stride length compared to those who are having a Stroke. The experimental results show that PD is bilateral and Stroke is unilateral. Patients with Stroke disease show a clear difference between affected and unaffected lower limb movements. In PD, symptoms of the disease may appear on both limbs.

Future extensions to this research can be suggested as follows. This study was limited to sagittal plane observations. Therefore a future work will be focused on gait deviations in frontal and transversal planes. Although the study used ankle, knee, and hip joint angles independently to identify gait deviations, gait pattern is a complicated process to study since the above three mentioned joints depend on each other. Considering this dependency, in the future, a suitable techniquecan be used todetect abnormalities.

\section{Compliance with ethical standards}

\section{Acknowledgments}

The authors are grateful to the hospital staff and the Department of Physics, the University of Sri Jayewardenepura for providing guidance and facilitation for data collection.

\section{Disclosure of conflict of interest}

There is no conflict of interest regarding the publication of this article.

\section{Statement of ethical approval}

For this research, the NHSL ethical clearance committee granted the ethical clearance.

\section{Statement of informed consent}

Informed consent was obtained from all individual participants included in the study.

\section{References}

[1] Shayo FK. (2019). Co-occurrence of risk factors for non-communicable diseases among in-school adolescents in Tanzania: an example of a low-income setting of sub-Saharan Africa for adolescence health policy actions. BMC Public Health, 19(1), 972.

[2] Dick FD, De Palma G, Ahmadi A, Scott NW, Prescott GJ, Bennett J, Semple S, Dick S, Counsell C, Mozzoni P and Haites N. (2007). Environmental risk factors for Parkinson's disease and parkinsonism: the Geoparkinson study. Occupational and Environmental Medicine, 64(10), 666-72.

[3] Sofuwa O, Nieuwboer A, Desloovere K, Willems A, Chavret F and Jonkers I. (2005). Quantitative Gait Analysis in Parkinson's Disease: Comparison with a Healthy Control Group. Archives of Physical Medicine and Rehabilitation, 86(5), 1007-1013.

[4] Morris ME, Iansek R, Matyas TA and Summers JJ. (1994). The pathogenesis of gait hypokinesia in Parkinson's disease. Brain, 117(5), 1169-81.

[5] Mazzoni P, Shabbott B and Cortés JC. (2012). Motor control abnormalities in Parkinson's disease. Cold Spring Harbor Perspectives in Medicine, 2(6), a009282. 
[6] Li S, Francisco GE and Zhou P. (2018). Post-stroke hemiplegic gait: new perspective and insights. Frontiers in physiology, 9, 1021.

[7] Blin 0, Ferrandez A and Serratrice G. (1990). Quantitative analysis of gait in Parkinson patients: increased variability of stride length. Journal of the Neurological Sciences, 98(1), 91-97.

[8] Kaczmarczyk K, Wit A, Krawczyk M andZaborski J. (2009). Gait classification in post-stroke patients using artificial neural networks. Gait \& Posture, 30(2), 207-210.

[9] Hodgins D. (2008). The importance of measuring human gait. Medical Device Technology, 19(5), 42-44.

[10] Pirker W and Katzenschlager R. (2017). Gait disorders in adults and the elderly. Wiener KlinischeWochenschrift, 129(3-4), 81-95.

[11] Thatrigoda DA and Rodrigo DS. (2014). Numerical Implementation of Fourier Transforms and Associated Problems. International Journal of Multidisciplinary Studies, 1(1).

[12] Wang W, Li K, Yue S, Yin C and Wei N. (2017). Associations between lower-limb muscle activation and knee flexion in post-stroke individuals: A study on the stance-to-swing phases of gait. Plos One, 12(9).

[13] Keloth, Viswanathan, Jelfs, Arjunan, Raghav and Kumar. (2019). Which Gait Parameters and Walking Patterns Show the Significant Differences Between Parkinson's Disease and Healthy Participants?. Biosensors, 9(2), 59.

\section{How to cite this article}

Dias KMGIU, Munasinghe A, Ravindra S, Rodrigo DS andSenanayake B. (2020). Spectral analysis of pathological gait characteristics of patients suffering from Parkinson disease and Strokes. World Journal of Advanced Research and Reviews, 6(3), 189-196. 